\title{
Pancreatic Neuroendocrine Tumors: a Modern Approach to Treatment
}

\author{
DOI: $10.17691 / \mathrm{stm} 2019.11 .3 .10$
}

Received February 12, 2019

D.M. Kuchin, MD, PhD, Oncology Surgeon, Oncology Department, Clinical Hospital No.11:

A.A. Seregin, Head of the Department of Endovascular X-Ray Diagnostic and Treatment Methods ${ }^{1 .}$

V.E. Zagainov, MD, DSc, Head of the Department of Intermediate Level Surgery and Transplantation; Chief Specialist in Surgery ${ }^{1}$

${ }^{1}$ Privolzhsky District Medical Center of Federal Medico-Biologic Agency of Russia, 14 llyinskaya St., Nizhny Novgorod, 603109, Russia;

2Privolzhsky Research Medical University, 10/1 Minin and Pozharsky Square, Nizhny Novgorod, 603005, Russia

The aim of the study was to evaluate efficacy of combined treatment for well-differentiated pancreatic neuroendocrine tumors according to the data provided by a specialized center.

Materials and Methods. Treatment of 40 patients with pancreatic neuroendocrine tumors was assessed. All of the patients underwent surgical removal of the primary tumor. Liver metastases (stage IV) were diagnosed in 14 patients, postoperative courses of hepatic artery chemoembolization were performed in 12 patients, and 10 of them had well-differentiated cancer.

Results. Good survival rates in patients with stage IV disease $(n=12)$ are comparable with those in the group of patients with stage III $(n=6)$ provided tumors are well-differentiated and courses of intra-arterial hepatic chemoembolization are given. In patients with poorly differentiated neuroendocrine tumors $(n=7)$, there was progression within a short time, but survival was less than 1-year after surgery despite active management.

Conclusion. To manage well-differentiated pancreatic neuroendocrine tumors, aggressive surgical approach should be used, despite the presence of metastatic lesions. In management of poorly differentiated neuroendocrine cancer, surgical approach proves to have unsatisfactory long-term results.

Key words: neuroendocrine tumor; pancreatectomy; hepatic artery chemoembolization.

\section{Introduction}

Neuroendocrine tumors (NETs) are a heterogeneous group of neoplasms that have biologically active properties and arise from neuroendocrine cells of the embryonic intestine. NETs are found in all organs that have neuroendocrine cells. Their most frequent location is the gastrointestinal tract and the pancreas, while the lungs come second [1]. The incidence of pancreatic and gastrointestinal NETs is low and scarcely reaches $2 \%$ among all cancers of these localizations $[2,3]$. Nevertheless, some authors believe every tenth detected pancreatic malignancy to be a neuroendocrine tumor [4]. In recent years, the frequency of diagnosing NETs has increased several times due to improvements in laboratory and instrumental diagnostic methods.

Neuroendocrine tumors are divided into functioning, or hormone-active, and non-functioning. Clinically manifested (functioning) lesions include insulinoma, glucagonoma, somatostatinoma, gastrinoma, vipoma, serotonin-producing tumor, ACTH-producing tumor. Detected NETs are most often non-functioning. The incidence of non-functioning pancreatic NETs reaches
$60-65 \%$ [5]. As a rule, their progression is asymptomatic. For this reason, in $59-80 \%$ of cases, the diagnosis is established when cancer process is advanced and distant metastases are detected or in the presence of regional tumor process.

Remarkably, there is pronounced heterogeneity in the group of patients with non-functioning NETs. Despite the late diagnosis, in $30-40 \%$ of cases, patients demonstrate quite satisfactory 5-year survival rate after treatment $[6,7]$. At the same time, another category of NET patients with a similar clinical picture survives even less than one year.

The degree of tumor differentiation is the main factor determining prognosis of survival and long-term outcomes of surgical and combined treatment. According to the 2010 WHO classification, there are two groups of tumors classified in terms of $\mathrm{Ki}-67$ proliferative activity index: well-differentiated (G1, G2), for which Ki-67 is $0-20 \%$, and poorly-differentiated (G3) with Ki-67 being more than $20 \%$. Patients with well-differentiated tumors demonstrate better survival rates than patients with poorly-differentiated ones [8]. Not only the degree of morphological tumor differentiation underlies significant differences in survival,

Corresponding author: Denis M. Kuchin, e-mail: pomc.kuchin@gmail.com 
but also greater sensitivity of well-differentiated tumors to chemotherapy and their generally less aggressive progression. Therefore, the main treatment modality for well-differentiated tumors $\mathrm{G} 1$ and $\mathrm{G} 2$ is active surgical management followed by adjuvant chemotherapy, even in the presence of distant metastases.

In addition to the degree of differentiation, long-term survival rate is also influenced by the extent of tumor process estimated by the TNM classification. There are two classifications of NETs: ENETS and AJCC. Their main difference is staging of the tumor. For example, according to the AJCC classification, stage I includes tumors T1 and T2: less than and more than $2 \mathrm{~cm}$ in size, localized within the pancreas. ENETS stage I includes only tumors smaller than $2 \mathrm{~cm}$. According to the AJCC classification, tumors spreading beyond the pancreas with lymph node metastases are staged as II, while ENETS stage II only includes tumors larger than $2 \mathrm{~cm}$, without the presence of lymph node metastases. ENETS stage III includes any tumors with lymph node metastases, while in the AJCC classification, this stage describes only tumors invading the celiac trunk or the superior mesenteric artery. Stage IV in both classifications categorizes patients with presence of distant metastases. In this study, we used the ENETS classification primarily due to its greater specificity in relation to the pancreas and staging effectiveness, which was demonstrated in one of the largest multicenter investigations [9].

To manage well-differentiated NETs staged III and IV, we used a combined method involving surgical removal of the primary tumor with subsequent hepatic artery chemoembolization (HACE).

The aim of the study was to evaluate efficacy of combined treatment for well-differentiated pancreatic neuroendocrine tumors according to the data provided by a specialized center.

\section{Materials and Methods}

A total of 404 patients with pancreatic tumors admitted between 2009 and 2017 were operated on at Privolzhsky District Medical Center of FMBA of Russia. Among them,

Table 1

Types of surgery performed based on the degree of tumor differentiation and disease stage

\begin{tabular}{lccc}
\hline $\begin{array}{c}\text { Disease stage/ } \\
\text { degree of tumor } \\
\text { differentiation }\end{array}$ & $\begin{array}{c}\text { Gastropancreato- } \\
\text { duodenal resection } \\
(n=22)\end{array}$ & $\begin{array}{c}\text { Distal } \\
\text { hemipancreatectomy } \\
(n=16)\end{array}$ & $\begin{array}{c}\text { Tumor } \\
\text { enucleation } \\
(n=2)\end{array}$ \\
\hline I, II/G1, G2 (n=15) & 9 & 4 & 2 \\
\hline I, II/G3 (n=1) & 1 & - & - \\
\hline III/G1, G2 (n=6) & 6 & - & - \\
III/G3 (n=4) & 3 & 1 & - \\
\hline IV/G1, G2 $(n=12)$ & 3 & 9 & - \\
\hline IV/G3 $(n=2)$ & - & 2 & - \\
\hline
\end{tabular}

40 persons $(10 \%$ of the total) had pancreatic NETs (13 males and 27 females). The mean age of patients was $49.0 \pm 5.9$ years. In 16 cases, the primary tumor was localized in the pancreatic body and (or) tail, and in 24 patients - in the pancreatic head.

Patients with non-functioning tumors ( $n=34,85 \%)$ had non-specific complaints: they had pain in the upper abdomen or signs of mechanical jaundice. Specific complaints such as dizziness, loss of consciousness, diarrhea, heartburn, nausea, vomiting were reported less often. Multiple liver metastases revealed by ultrasound were documented as the cause of admission in 2 cases.

Functioning tumors $(n=6,15 \%)$ had a bright specific clinical picture, which was the reason for early contact with healthcare services. Interestingly, no patient with a hormone-functioning tumor had distant metastases at the time of diagnosis. In patients with functioning NETs, insulinoma was revealed in four cases, gastrinoma in one case and one patient was diagnosed with recurrent vipoma with high hormonal activity.

The study complies with the Declaration of Helsinki (2013) and was performed following approval by the Ethics Committee of Privolzhsky Research Medical University. Written informed consent was obtained from every patient.

Apart from biochemical and general blood tests, laboratory studies included assessment of insulin level, C-peptide, gastrin, and chromogranin A, vasoactive intestinal peptide. Instrumental diagnostics consisted of abdominal ultrasound, fibrogastroduodenoscopy (FGDS), colonoscopy, abdominal CT scan with intravenous contrast or MRI. All patients with metastatic lesions underwent a fine-needle percutaneous biopsy of the metastatic focus in the liver under ultrasound guidance at the preoperative stage. Morphological study of the tumor was carried out using light microscopy followed by immunohistochemical examination and evaluation of such markers as chromogranin $A$, index Ki-67, synaptophysin.

All patients were operated on after follow-up examination. The purpose of the surgery was to remove the primary tumor. Corporocaudal distal pancreatectomy was performed in 16 patients, gastropancreatoduodenal resection - in 22, enucleation of the tumor -2 (Table 1).

Well-differentiated NETs (G1, G2) were diagnosed in 33 patients $(82.5 \%)$, the remaining 7 patients had poorly-differentiated tumors.

In the group of patients with welldifferentiated NETs, 15 persons had resectable tumor within the organ (stage I, II according to the TNM classification), 6 patients were diagnosed with stage III. Liver metastases (stage IV) were revealed in 12 patients, 10 of them had bilobate metastases. In the group of patients with poorly-differentiated NETs, one patient was reported to have stage II, stage III 
Table 2

Distribution of operated patients (abs. number/\%)

\begin{tabular}{lccc}
\hline Degree of tumor differentiation & $\begin{array}{c}\text { Stages } \\
\text { I and II }\end{array}$ & $\begin{array}{c}\text { Stage } \\
\text { III }\end{array}$ & $\begin{array}{c}\text { Stage } \\
\text { IV }\end{array}$ \\
Well-differentiated & $15 / 37.5$ & $6 / 15$ & $12 / 30$ \\
\hline Poorly-differentiated & $1 / 2.5$ & $4 / 10$ & $2 / 5$ \\
\hline
\end{tabular}

was diagnosed in 4 patients. Stage IV was revealed in 2 patients due to bilobate metastatic liver disease (see Table 1, Table 2).

In the postoperative period, all patients with metastatic liver disease underwent HACE. It was carried out as a therapeutic sequence with 5-fluorouracil, doxorubicin, gemcitabine. After two HACE courses, one patient underwent atypical resection of segment 2 of the liver with subsequent evaluation of therapeutic pathomorphosis.

\section{Results and Discussion}

Postoperative complications were assessed according to the Clavien-Dindo classification. Complications of grades II and III were reported in 5 patients. Two of them had pancreatic fistula of grade B, 1 patient had intra-abdominal bleeding, 1 had high small-bowel obstruction, 1 had gastrointestinal bleeding in the area of gastroenteroanastomosis. Thus, the total proportion of complications amounted to $12.5 \%$, the proportion of repeated surgeries $-5 \%$. There were no fatal outcomes among patients of this group in the early postoperative period.

Efficacy of intra-arterial chemoembolization for NET with liver metastases was first shown by $\mathrm{Ho}$ et al. in 2007 [10]. They performed HACE in 46 patients and achieved a significant increase in median survival up to 42 months.

Development of modern endovascular technologies offers new possibilities to manage patients with disseminated NETs, which allows achieving good results of overall and recurrence-free survival [4]. We performed intra-arterial chemoembolization in 12 out of 14 patients with metastatic liver disease after removal of the primary tumor (two patients with a low degree of tumor differentiation).

Immediately in the course of intra-arterial chemoembolization, 5 patients $(42 \%)$ were diagnosed with clinical manifestations such as feeling short of breath, bronchospasm, abdominal pain, 4 patients (33\%) had postembolization syndrome manifested by an increase in body temperature and a sensation of heaviness in the right hypochondrium. All side effects of HACE were successfully treated conservatively. In one case, after a course of chemoembolization, the patient developed acute liver failure, which was successfully managed conservatively. However, follow-up observations revealed multiple liver abscesses in both lobes, and 2 months after HACE, there was a fatal outcome following septic complications.

Strosberg et al. [11] and Yang et al. [12] classified patients with NETs stage I and stage II into one group. This was determined by lack of differences in the longterm survival of these patients. In our study, 15 patients with well-differentiated resectable tumors staged I and II, according to the ENETS classification, are alive, the follow-up period being 10 to 62 months.

In the group of patients with stage III tumors and differentiation degree $\mathrm{G} 1, \mathrm{G} 2$, three out of 6 patients are alive, the follow-up period ranging from 6 to 36 months, three patients died after 24,28 , and 36 months.

Thus, the expected response to treatment for NETs significantly depends on the disease stage.

In 10 cases of established well-differentiated pancreatic NETs with metastatic lesions to the liver, our first step was removing the primary tumor, the second - a course of HACE treatment. A pronounced positive effect was obtained in 5 out of 10 patients who showed complete disappearance of lesions in the liver, no additional therapy was received by any of them. All of the patients are alive, there are no signs of recurrence, the follow-up period being 9 to 48 months. The remaining 5 patients showed positive dynamics in the form of reduced number and size of lesions or process stabilization. Now, 2 of these patients are followed up during the period of 8 to 28 months. The remaining 3 patients died 20, 24, 34 months after surgical treatment. All 5 patients of this group received chemotherapy with somatostatin analogues in combination with HACE courses. All 10 patients had bilobate metastatic liver disease, but maximum size of lesions in the liver of the first 5 patients with a pronounced positive effect was no more than $35 \mathrm{~mm}$, in the other 5 patients it was within $70 \mathrm{~mm}$. Two patients underwent only removal of the primary tumor, followed by chemotherapy with platinum drugs (2011), in both cases, fatal outcome was reported after 16 and 23 months (Figures 1, 2).

Thus, patients with stage I and II well-differentiated tumors show a tendency to better long-term survival rates compared to patients with disease stages III and IV. Our findings are consistent with the results of other studies: the five-year survival rate for patients with stage I, II NETs is 2.5 times higher than for patients with stage III tumor [12].

Only one out of 7 patients diagnosed with primary resectable low-differentiated NET died from cancer progression 8 months after the operation. Four patients with disease stage III and differentiation degree G3 failed to survive 7 months. Two patients with low-differentiated tumor and metastatic liver disease died within the first four months after surgery (Table 3).

Thus, given the relatively favorable prognosis of pancreatic NETs, we consider aggressive complex treatment of patients with disease stages III and IV and high degree of tumor differentiation to be appropriate 

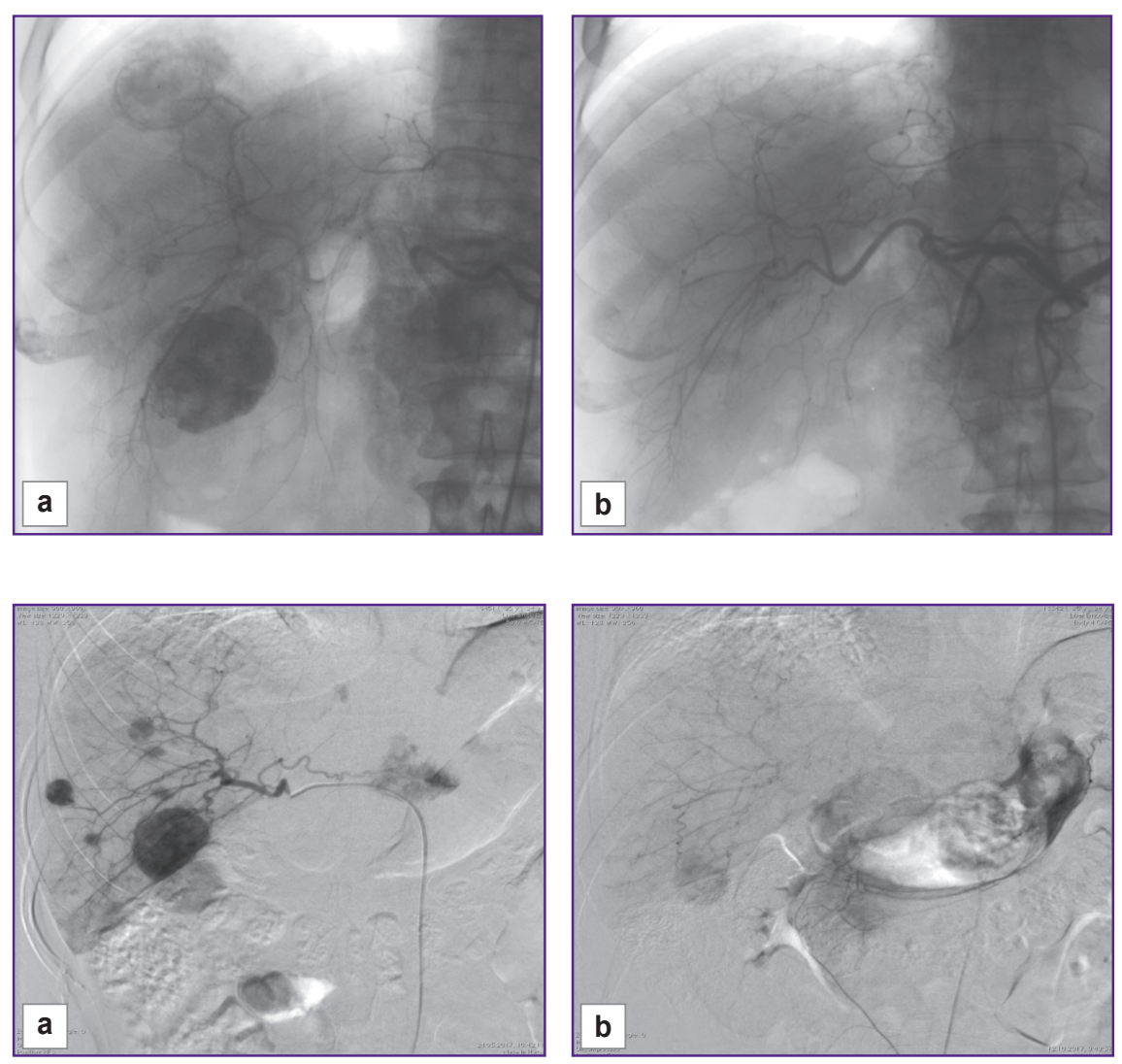

Figure 1. Angiogram of patient $\mathrm{N}$., 36 years, after pancreatoduodenal resection for metastatic welldifferentiated neuroendocrine tumor: (a) before the first course of hepatic artery chemoembolization; (b) after three courses of hepatic artery chemoembolization with doxorubicin

Figure 2. Angiogram of patient V., 44 years, after distal pancreatectomy for metastatic well-differentiated neuroendocrine tumor:

(a) during the first course of hepatic artery chemoembolization; (b) during the fourth course of hepatic artery chemoembolization with doxorubicin
Table 3

Long-term survival results (abs. number/\%)

\begin{tabular}{lcc}
\hline \multicolumn{1}{c}{$\begin{array}{c}\text { Disease stage/degree } \\
\text { of tumor differentiation }\end{array}$} & $\begin{array}{c}\text { Died } \\
\text { within 1 year }\end{array}$ & $\begin{array}{c}\text { Died } \\
\text { within } 3 \text { years }\end{array}$ \\
\hline I, II/G1, G2 $(n=15)$ & 0 & 0 \\
\hline I, II/G3 $(n=1)$ & $1 / 100$ & - \\
\hline III/G1, G2 $(n=6)$ & 0 & $3 / 50$ \\
\hline III/G3 $(n=4)$ & $4 / 100$ & - \\
\hline IV/G1, G2 (n=12) & 0 & $5 / 41.6$ \\
\hline IV/G3 $(n=2)$ & $2 / 100$ & - \\
\hline
\end{tabular}

$[13,14]$. It is necessary to take into consideration not only radical but also conditionally radical and even cytoreductive surgery for this group of patients, providing additional specific treatment (HACE, adjuvant polychemotherapy), in contrast to patients with lowdifferentiated NETs. Our findings are consistent with the data of Granov et al. [8] confirming that more than $82 \%$ of patients with metastatic forms G1, G2 of pancreatic NETs survive 5 to 119 months.

We believe that after removal of the primary tumor, isolated use of HACE in metastatic liver disease is efficacious and able to improve long-term survival compared to the survival rates of patients without distant metastases. The use of somatostatin analogues is appropriate for adjuvant treatment in this group of patients only after determining the expression of somatostatin receptors in pancreatic NET $[15,16]$.

\section{Conclusion}

Factors affecting the prognosis of the disease in neuroendocrine tumors are the stage of the disease and proliferative activity of tumor cells (Ki-67 index). Patients with well-differentiated stage I and stage II tumors have good long-term survival results and require no adjuvant treatment if radical surgery is performed. The use of hepatic artery chemoembolization in patients with well-differentiated neuroendocrine tumors accompanied by liver metastases is likely to improve long-term survival. To manage well-differentiated pancreatic neuroendocrine tumors, aggressive surgical approach is appropriate even in presence of multiple metastatic lesions. In management of poorly differentiated neuroendocrine cancer, surgical approach proves to have unsatisfactory long-term results, which necessitates further development of neoadjuvant chemotherapy protocols.

Study funding. This study was not supported by any financial sources.

Conflict of interests. The authors have no conflict of interests to disclose. 


\section{References}

1. Onkologiya. Klinicheskie rekomendatsii [Oncology. Clinical guidelines]. Pod red. Davydova M.I. [Davydov M.I. (editor)]. Moscow: Izdatel'skaya gruppa RONTs; 2015; 680 p.

2. Chen C., Yi X., He Y. Gastro-entero-pancreatic neuroendocrine tumor (GEP-Nets): a review. J Gastrointest Dig Syst 2013; 3(5): 2161-2169, https://doi.org/10.4172/2161069x.1000154.

3. Lairmore T.C., Quinn C.E., Martinez M.J. Neuroendocrine tumors of the pancreas: molecular pathogenesis and current surgical management. Transl Gastrointest Cancer 2014; 3(1): 29-43.

4. McKenna L.R., Edil B.H. Update on pancreatic neuroendocrine tumors. Gland Surg 2014; 3(4): 258-275.

5. Yao J.C., Hassan M., Phan A., Dagohoy C., Leary C., Mares J.E., Abdalla E.K., Fleming J.B., Vauthey J.N., Rashid A., Evans D.B. One hundred years after "carcinoid": epidemiology of and prognostic factors for neuroendocrine tumors in 35,825 cases in the United States. J Clin Oncol 2008; 26(18): 3063-3072, https://doi.org/10.1200/ jco.2007.15.4377.

6. Simonenko V.B., Dulin P.A., Makanin M.A. Kartsinoidy i neyroendokrinnye opukholi [Carcinoid and neuroendocrine tumors]. Moscow: Meditsina; 2008; 176 p.

7. Mignon M. Natural history of neuroendocrine enteropancreatic tumors. Digestion 2000; 62(1): 51-58, https:// doi.org/10.1159/000051856.

8. Granov D.A., Pavlovskiy A.V., Popov S.A., Polikarpov A.A., Moiseenko V.E. Combined treatment of patients with pancreatic neuroendocrine carcinomas. Annaly khirurgicheskoy gepatologii 2016; 21(1): 32-37, https://doi. org/10.16931/1995-5464.2016132-37.

9. Glotov A.V., Kalinin D.V., Smirnov A.V., Kriger A.G. Staging of pancreatic neuroendocrine tumor (literature review).
Annaly khirurgicheskoy gepatologii 2017; 22(3): 70-76, https:// doi.org/10.16931/1995-5464.2017370-76.

10. Ho A.S., Picus J., Darcy M.D., Tan B., Gould J.E., Pilgram T.K., Brown D.B. Long-term outcome after chemoembolization and embolization of hepatic metastatic lesions from neuroendocrine tumors. AJR Am J Roentgenol 2007; 188(5): 1201-1207, https://doi.org/10.2214/ajr.06.0933.

11. Strosberg J.R., Cheema A., Weber J., Han G., Coppola D., Kvols L.K. Prognostic validity of a novel American Joint Committee on Cancer Staging Classification for pancreatic neuroendocrine tumors. J Clin Oncol 2011; 29(22): 3044-3049, https://doi.org/10.1200/jco.2011.35.1817.

12. Yang M., Zeng L., Zhang Y., Wang W.G., Wang L., Ke N.W., Liu X.B., Tian B.L. TNM staging of pancreatic neuroendocrine tumors. Medicine 2015; 94(12): e660, https:// doi.org/10.1097/md.0000000000000660.

13. Lesurtel M., Nagorney D.M., Mazzaferro V., Jensen R.T., Poston G.J. When should a liver resection be performed in patients with liver metastases from neuroendocrine tumours? A systematic review with practice recommendations. HPB 2015; 17(1): 17-22, https://doi.org/10.1111/hpb.12225.

14. Rossi R.E., Massironi S., Conte D., Peracchi M. Therapy for metastatic pancreatic neuroendocrine tumors. Ann Transl Med 2014; 2(1): 8.

15. Gurevich L.E., Korsakova N.A., Voronkova I.A., Ashevskaya V.E., Titov A.G., Kogoniya L.M., Egorov A.V., Britvin T.A., Vasil'ev I.A. Immunohistochemical determination of expression of somatostatin receptors types 1, 2A, 3 and 5 in neuroendocrine tumors of various localization and grade. Al'manah kliniceskoj mediciny 2016; 44(4): 378-390, https:// doi.org/10.18786/2072-0505-2016-44-4-378-390.

16. Laskaratos F.M., Caplin M. Treatment challenges in and outside a network setting: gastrointestinal neuroendocrine tumours. Eur J Surg Oncol 2019; 45(1): 52-59, https://doi. org/10.1016/j.ejso.2018.03.012. 